\title{
Marketing deontológico: herramienta para la sostenibilidad ambiental
}

\author{
Edwin VÁsquez Erazo* \\ Juan Sebastian Vásquez Álvarez** \\ Jessica VÁsquez Álvarez $* * *$
}

\begin{abstract}
RESUMEN
El objetivo de esta investigación fue desarrollar un modelo conceptual de marketing que aporte a la responsabilidad social ambiental de empresas y consumidores para disminuir la contaminación, contribuyendo a mejorar la calidad de vida, para lo cual se realizó primero una investigación con fuentes secundarias que permitieran constatar la existencia de indicios de procesos de contaminación en los diferentes segmentos identificados de tal forma que puedan orientar la propuesta, del análisis efectuado. Se obtuvieron los elementos fundamentales para la construcción del modelo de gestión. Como resultado se constató que el papel del marketing deontológico ha mejorado la conducta, los hábitos de los consumidores y la forma de producción de los empresarios con relación al cuidado y protección del ambiente. De igual manera se establecen nuevas dimensiones con relación a las variables del marketing mix, incorporando tres elementos, personas, protección, producción limpia y se identifican estrategias que aporten al desarrollo sostenible.
\end{abstract}

Palabras clave: marketing deontológico, marketing ético, Marketing social, marketing ambiental

The DEONTOLOGICAL MARKETING: TOOL FOR ENVIRONMENTAL SUSTAINABILITY APPLIED TO AMBATO CITY OF ECUADOR

\section{ABSTRACT}

The objective of this research was develop a conceptual model of marketing aports to the environmental social responsability of enterprises and consumers to reduce the pollution, contributing to make better lifestyle, It was first a research with secondary sources that would verify the existence of signs of pollution processes in diferente segments identified so that they can guide the proposal, the analysis made. The fundamental elements for the construction of the management model were. As a result it was found that the role of deontological marketing has improved behavior, consumer habits and the way of production of employers in relation to the care and protection of the environment. Similarly, new dimensions are established in relation to the marketing mix variables, incorporating three elements, production, protection, clean and identify strategies that contribute to sustainable development.

Keywords: deontological marketing, ethic marketing, social marketing, environmental marketing

\section{INTRODUCCIÓN}

¿Por qué marketing deontológico con responsabilidad social ambiental?

La constante destrucción y contaminación que día a día sufre nuestro planeta ha hecho que la mayor parte de la sociedad tome conciencia de la problemática ambiental, por lo tanto, es posible conseguir al mismo tiempo la satisfacción de las necesidades de los consumidores, la maximización de los beneficios de la empresa y la minimización de los impactos negativos sobre el medio ambiente

El marketing deontológico, está dentro del campo amplio del Marketing Social, no busca rentabilidad económica, sino que integra todos los subsistemas sociales para lograr la concientización y el cambio de conducta de empresas y consumidores para conseguir una finalidad muy importante el cuidado y la protección del ambiente y con ello garantizar los recursos para el futuro.

Desde la mirada epistemológica, el marketing se fundamente en la realidad para construir conocimiento, y que se valore como verdadero los modelos que demuestran funcionar u obtener resultados en la realidad, desde una visión pragmática en cambio implica en efecto una cierta manera de entender la estructura de la experiencia, otro enfoque importante en la medida de la innovación de marketing tiene que ver con la complejidad, gestión de un área específica en la organización, nace de la voluntad de los que la conforman, sin desligarse del resto de los subsistemas que integran la institución y estos con relación al entorno, produciéndose una vinculación entre los sujetos y el objeto.

En consecuencia, para conseguir el desarrollo sostenible, las empresas deben gestionar sus procesos en base a políticas de producción más limpia y responsabilidad social ambiental, de tal manera que no se comprometa la capacidad de satisfacer las necesidades futuras de las próximas generaciones. De igual forma, el marketing deontológico para aportar a la sostenibilidad tiene algunas acciones: concientización medioambiental a

* Magister en Dirección de Empresas, Funcionario de la Universidad Técnica de Cotopaxi, cursant del doctorado en Gestión de Empresas en la Universidad San Marcos Facultad de Ingeniería Industrial. E-mail: edwinvasquez11@hotmail.com

* Ingeniero en Administración de Empresas, Gerente Administrativo, Empresa DISOLVAS E-mail: juansebas9800@hotmail.com

* Magister en Docencia Universitaria, Funcionario de la Universidad Técnica de Cotopaxi. E-mail: jessitavasquez@hotmail.com 
los empresarios, consumidores y demás grupos de interés, definir las variables del marketing, implementar incorporar el factor medioambiental, crear una cultura que trascienda las fronteras de la empresa y de los stakeholders.

En cuanto al problema a solucionar se plantea ¿Cómo incrementar la responsabilidad social ambiental de empresas y consumidores de la Ciudad de Ambato - Ecuador? Con la finalidad de asumir una posición positiva ante los crecientes problemas que están afectando a la humanidad como la contaminación ambiental y una sociedad de consumo que cada día tiene menos conciencia social. Con relación al marketing y la contaminación ambiental, algunos autores manifiestan:

(Stanton, 2000), en su marketing ético, plantea que: "Algunas empresas generar productos adulterados, productos defectuosos, productos $y$ servicios inseguros, presentan productos caducados y lo que es más grave una gran cantidad de envases y embalajes que generalmente van a la basura produciendo contaminación al medio ambiente"; refiriéndose al tema (Cannon, 1994), afirma: "Las empresas, al no tener producciones limpias no actúan con responsabilidad social generando un gran impacto negativo en la sociedad". Finalmente con relación a los consumidores: "Las personas somos constantemente amenazadas por publicidades que generan las empresas, diariamente escuchamos que la televisión nos manipula, que el público entendido como una masa amorfa se enajena, se frustra, siente deseos de emular a los héroes de los programas" (Garcia Roca, 1994).

Por otro lado (Molina, 2010); Algunas personas, no somos socialmente responsables, no tenemos cultura social y arrojamos los desperdicios por todo lado sin tener en cuenta el tipo de material que se está botando, es decir no existe una cultura de reciclaje y peor aún de cuidar nuestro planeta; refiriéndose a los consumidores (sociedad), (Albardiaz, 1998) manifiesta que: "La compra ecológica, no ha crecido al mismo ritmo que la sensibilización medioambiental de la sociedad, producción una brecha entre los requerimientos de la sociedad moderna y los consumidores, cada vez compramos más productos con envases no degradables, o que no se los pueda reutilizar, contribuyendo a la acumulación de desperdicios en los hogares, por otra parte la dificultad de encontrar productos ecológicos, es referida como freno principalmente por personas cuyas edades oscilan entre 35 y 49 año".

La metodología del estudio planteado, se realiza a través de una investigación exploratoria tipo cualitativa de corte descriptivo concluyente, cuyo objetivo es la descripción y caracterización de las acciones que el marketing deontológico desarrollará para cambiar la manera de pensar y actuar de las personas.

El marketing deontológico es una rama del marketing social, cuyo objetivo no es promocionar un producto o servicio sino diseñar programas donde se incentive a un determinado grupo de individuos a adoptar ciertas ideas o a cambiar alguna actitud o comportamiento (Vázquez y Trespalacios, 1997).

El Modelo planteado de Marketing Deontológico, tiene como finalidad generar estrategias que orienten a los empresarios y consumidores a buscar formas responsables sobre el cuidado y la protección del medio ambiente, en el que se integran elementos fundamentales como las tradicionales 4 Ps del marketing mix y se incorpora 3 Ps muy importantes que se articulan a un conjunto de estrategias que lleven a la consecución de la Sostenibilidad Ambiental como son: Generación de mercados estratégicos verdes, Producción limpia y consumo sostenible, Inclusión de la variable sostenibilidad en cadenas productivas.

El tema de la contaminación ambiental, en los actuales momentos, ya no es exclusivo de especialistas o investigadores de ecología y medioambiente, es algo que nos atañe a todos y que nos debería de empezar a interesar pues TODOS somos responsables del daño que estamos causando al planeta.

\section{MATERIALES Y MÉTODOS}

El proceso investigativo nace como una idea de contribuir al cuidado y preservación del ambiente. La contaminación ambiental, es una de las preocupaciones muy grandes a nivel mundial y tiene relación con situaciones ocasionadas por actividades, procesos o comportamientos humanos, económicos, culturales y políticos, ocasionando impactos negativos sobre el ambiente que afectan a todo el mundo, teniendo como fundamento el Objetivo N.7 del Desarrollo del milenio, "Garantizar la sostenibilidad del medio ambiente" y de manera particular el literal 4 "Apoyar la formulación de estrategias que vinculen el tema ambiental con el comercio" (ONU, 2000) y el Plan de desarrollo del Ecuador, objetivo 7, "Garantizar los derechos de la naturaleza y promover la sostenibilidad ambiental territorial y global" y de manera particular con la política 7.8 Controlar y Mitigar la Contaminación Ambiental (Controlar y Mitigar la Contaminación Ambiental.(Senplades 2013-2017). 
Tabla 1. Resultados de Frecuencia de respuestas sector empresarial

\begin{tabular}{|c|c|c|c|c|c|c|c|}
\hline \multirow{2}{*}{ N. ${ }^{\circ}$} & \multirow{2}{*}{ CUESTIONARIO } & \multicolumn{5}{|c|}{$\begin{array}{l}\text { SEGMENTO EMPRESARIAL DE AMBATO- } \\
\text { ECUADOR }\end{array}$} & \multirow{2}{*}{$\begin{array}{l}\text { TOTAL DE } \\
\text { ENCUESTADOS }\end{array}$} \\
\hline & & Nunca & Rara Vez & $\begin{array}{l}\text { Algunas } \\
\text { Veces }\end{array}$ & $\begin{array}{l}\text { Casi } \\
\text { Siempre }\end{array}$ & Siempre & \\
\hline 1 & $\begin{array}{l}\text { ¿Aplica su empresa un Plan de } \\
\text { Marketing Responsable? }\end{array}$ & 190 & 10 & 19 & 14 & 6 & 239 \\
\hline 2 & $\begin{array}{l}\text { ¿Su empresa ha producido } \\
\text { productos defectuosos? }\end{array}$ & 239 & 0 & 0 & 0 & 0 & 239 \\
\hline 3 & $\begin{array}{l}\text { ¿Sus productos tienen envases } \\
\text { biodegradables? }\end{array}$ & 86 & 43 & 43 & 25 & 42 & 239 \\
\hline 4 & $\begin{array}{l}\text { ¿Cumple las normas de } \\
\text { etiquetado? }\end{array}$ & 0 & 9 & 35 & 96 & 99 & 239 \\
\hline 5 & $\begin{array}{l}\text { ¿Tiene su etiqueta orientación } \\
\text { ambiental al consumidor? }\end{array}$ & 87 & 35 & 32 & 43 & 42 & 239 \\
\hline 6 & $\begin{array}{l}\text { ¿Considera que la publicidad } \\
\text { comercial de su empresa induce } \\
\text { al cuidado y protección del } \\
\text { ambiente? } \\
\end{array}$ & 109 & 48 & 43 & 18 & 21 & 239 \\
\hline 7 & $\begin{array}{l}\text { ¿Se aplica políticas de reducción } \\
\text { del CO2? }\end{array}$ & 125 & 36 & 15 & 22 & 41 & 239 \\
\hline 8 & $\begin{array}{l}\text { ¿Se aplica políticas de reducción } \\
\text { de Residuos sólidos? }\end{array}$ & 108 & 32 & 0 & 60 & 39 & 239 \\
\hline 9 & $\begin{array}{l}\text { ¿Produce su empresa desechos } \\
\text { contaminantes? }\end{array}$ & 218 & 4 & 6 & 5 & 6 & 239 \\
\hline 10 & $\begin{array}{l}\text { ¿Genera su empresa algún tipo } \\
\text { de desperdicios? }\end{array}$ & 0 & 9 & 48 & 91 & 91 & 239 \\
\hline 11 & $\begin{array}{l}\text { ¿Aplica su empresa campañas } \\
\text { de responsabilidad Social? }\end{array}$ & 102 & 33 & 33 & 43 & 28 & 239 \\
\hline 12 & $\begin{array}{l}\text { ¿La publicidad comercial, } \\
\text { orienta sobre beneficios de los } \\
\text { productos? }\end{array}$ & 33 & 18 & 19 & 68 & 101 & 239 \\
\hline 13 & $\begin{array}{l}\text { ¿Utiliza su empresa políticas de } \\
\text { producción más limpia? }\end{array}$ & 100 & 30 & 45 & 30 & 34 & 239 \\
\hline
\end{tabular}

Se toma como punto de partida las definiciones de: (Kotler 1999) "El marketing social es un proceso de aplicación de técnicas, que comprenden: la investigación, análisis, planificación, diseño, ejecución, control y evaluación de programas; que tienen como objetivo, promover un cambio social favorable, que esté orientado a que sea aceptada o modificada, una determinada idea o práctica en uno o más grupos de destinatarios".

(Kotler y Zaltman 1975) define el marketing social como "el diseño, implantación y control de programas que buscan incrementar la aceptación de una idea o causa social en determinados grupos objetivo" 
El proceso de indagación se desarrolló partiendo del análisis bibliográfico con relación al marketing, marketing social, la responsabilidad social ambiental y la contaminación que contribuyan a tener elementos científicos para la generación de un nuevo conocimiento. Con la finalidad de contar con datos específicos relacionadas al problema de contaminación y responsabilidad social ambiental se realizó una investigación previa, tomando como punto de partida dos segmentos importantes uno relacionado al sector de consumidores, representados por: (amas de casa, estudiantes universitarios, profesionales y empleados públicos), y el otro segmento el empresarial compuesto por: (Metalmecánicas, Textil, Alimentarias).

El estudio planteado, se realiza a través de una investigación exploratoria tipo cualitativa de corte descriptivo concluyente, cuyo objetivo es la descripción y caracterización de las acciones que el marketing deontológico debe desarrollar para cambiar la manera de pensar y actuar de las personas, las empresas y los consumidores de la ciudad de Ambato - Ecuador, mediante, el estudio correlacional explicativo probabilístico, para establecer las relaciones entre variables y construir un conjunto de acciones generando un modelo orientado a incrementar la responsabilidad social ambiental de empresas y consumidores. Se tomó una muestra 239 empresas a las que se les aplico una encuesta considerando los siguientes escenarios: marketing, responsabilidad social ambiental, producción limpia y contaminación donde se obtuvo los siguientes resultados:

Con relación a los datos obtenidos de la encuesta se realizan las siguientes apreciaciones: La totalidad de empresas encuestadas generan algún tipo de desperdicios, existe Limitada aplicación de políticas de producción limpia, Muy pocas empresas cuentan con campañas para cuidar y proteger el ambiente, En un menor grado las empresas realizan el manejo de desechos sólidos, Algunos de sus envases no son biodegradables, en cuanto a las etiquetas no se encuentra orientación sobre la protección del ambiente, se evidencia que la mayoría de las empresas no cuentan con un plan de marketing.

Un segundo segmento analizado está relacionado con los consumidores. Se tomó la población total de habitantes 329856 (INEC 2010) y de los cuales se extrajo una muestra representativa de 384 a los que se les aplico la encuesta para obtener datos relacionados con tres áreas: marketing, responsabilidad social ambiental y contaminación.

Se puede notar que Los consumidores de la ciudad de Ambato- Ecuador no son compradores ecológicos, Muy pocos tienen precaución de revisar las etiquetas de sus envases, La mayor parte de ellos botan sus envases a la basura por lo que no existe una cultura de reciclaje, la publicidad sigue siendo el motor que incide a la hora de comprar. Estos elementos aportan para la construcción de estrategias de marketing.

El tipo de investigación aplicado, es de campo, mediante el estudio correlacional explicativo probabilístico, por cuanto nos permitirá establecer las relaciones entre variables dependientes e independientes, es decir se estudiaría la correlación existente entre los dos constructos sociales: marketing y responsabilidad social ambiental.

Otro elemento fundamental está relacionado a la actividad comercial es decir la relación de oferta y demanda, para lo que se es necesario tener muy claro el concepto de consumo sostenible, que se refiere al uso de servicios y productos que responden a las necesidades básicas y ofrecen una mejor calidad de vida, al mismo tiempo que minimizan el uso de recursos naturales y materiales tóxicos, así como la emisión de desechos y contaminantes durante el ciclo de vida del servicio o producto, sin poner en peligro las necesidades de futuras generaciones. ("PNUMA 1995)

\section{RESULTADOS}

Desde un enfoque filosófico del marketing social, en la que muestra cómo crear sistemas eficaces de producción y por consiguiente mejorar la rentabilidad de los negocios, entendiendo que estos son un subsistema social que cumple el papel económico y social. Por lo tanto, una empresa debe operar de modo que haga posible producir beneficios para la sociedad y al mismo tiempo producir beneficios para la propia organización]. (Braidot, 1996).

"En la actualidad, la producción, el consumo y la responsabilidad social ambiental, funcionan por separado. El objetivo del marketing es entonces integrar estos elementos.

\section{El marketing deontológico como herramienta para la sostenibilidad social ambiental.}

Para (Bentham 1828), la deontología se aplica fundamentalmente al ámbito de la moral, es decir, a aquellas conductas del ser humano que no forman parte de las hipótesis normativas del derecho vigente, aquellas acciones que no están sometidas al control de la legislación pública. Entonces el termino deontología, hace referencia, de manera general, al estudio o la ciencia de los deberes u obligaciones morales; por lo tanto El objeto de 
Tabla 2. Resultados de Frecuencia de respuestas sector empresarial

\begin{tabular}{|c|c|c|c|c|c|c|c|}
\hline \multirow{2}{*}{ N. ${ }^{\circ}$} & \multirow{2}{*}{ CUESTIONARIO } & \multicolumn{5}{|c|}{$\begin{array}{c}\text { SEGMENTO CONSUMIDORES DE AMBATO- } \\
\text { ECUADOR }\end{array}$} & \multirow{2}{*}{$\begin{array}{l}\text { TOTAL DE } \\
\text { ENCUESTADOS }\end{array}$} \\
\hline & & Nunca & Rara Vez & $\begin{array}{c}\text { Algunas } \\
\text { Veces }\end{array}$ & $\begin{array}{c}\text { Casi } \\
\text { Siempre } \\
\end{array}$ & Siempre & \\
\hline 1 & ¿Es comprador ecológico? & 95 & 78 & 62 & 60 & 89 & 384 \\
\hline 2 & $\begin{array}{l}\text { ¿En sus compras ha encontrado } \\
\text { productos en mal estado? }\end{array}$ & 213 & 100 & 48 & 11 & 12 & 384 \\
\hline 3 & $\begin{array}{l}\text { ¿Sus envases van a para a la } \\
\text { basura? }\end{array}$ & 22 & 42 & 71 & 110 & 139 & 384 \\
\hline 4 & $\begin{array}{l}\text { ¿Realiza un proceso de reciclaje } \\
\text { con sus envases? }\end{array}$ & 121 & 60 & 63 & 61 & 79 & 384 \\
\hline 5 & ¿Reutiliza sus envases? & 98 & 74 & 61 & 61 & 90 & 384 \\
\hline 6 & $\begin{array}{l}\text { ¿Puede usted reconocer un } \\
\text { envase biodegradable? }\end{array}$ & 56 & 64 & 58 & 68 & 138 & 384 \\
\hline 7 & $\begin{array}{l}\text { ¿Un precio Bajo de compra } \\
\text { le indica una mala calidad del } \\
\text { producto? }\end{array}$ & 72 & 39 & 63 & 85 & 125 & 384 \\
\hline 8 & $\begin{array}{l}\text { ¿Puede identificar la información } \\
\text { Tipo Semáforo en las etiquetas de } \\
\text { sus productos? (Ecuador) }\end{array}$ & 22 & 21 & 46 & 107 & 188 & 384 \\
\hline 9 & $\begin{array}{l}\text { ¿Revisa Ud. La etiqueta cuando } \\
\text { adquiere productos? }\end{array}$ & 109 & 71 & 65 & 63 & 76 & 384 \\
\hline 10 & $\begin{array}{l}\text { ¿Controla la caducidad de su } \\
\text { producto? }\end{array}$ & 47 & 50 & 76 & 91 & 120 & 384 \\
\hline 11 & $\begin{array}{l}\text { ¿Encuentra en la etiqueta } \\
\text { información Ambiental? }\end{array}$ & 261 & 55 & 34 & 17 & 17 & 384 \\
\hline 12 & $\begin{array}{l}\text { ¿Considera que la publicidad } \\
\text { comercial de los medios de } \\
\text { información inducen al cuidad y } \\
\text { protección ambiental? }\end{array}$ & 183 & 55 & 80 & 33 & 33 & 384 \\
\hline 13 & $\begin{array}{l}\text { ¿Incide la publicidad en sus } \\
\text { decisiones de compra? }\end{array}$ & 43 & 42 & 107 & 101 & 91 & 384 \\
\hline
\end{tabular}

estudio del marketing deontológico son los fundamentos del deber y las normas morales que rigen al marketing y su conjunto de operaciones.

El principal razonamiento de la dimensión social del marketing se tiene en el hecho de que las empresas existen con un propósito: servir a la sociedad, debiendo tener contenido ético y social sus prácticas de marketing-mix. Por otro lado desde una mirada epistemológica, el marketing se fundamenta en la realidad para construir conocimiento y que su valor dependerá de los resultados que se logren en la realidad.

Dentro del Modelo Conceptual de Marketing Deontológico, se tiene como propósito orientar a las empresas y consumidores para que sus procesos de oferta y demanda tengan formas responsables de actuación con relación al cuidado y la protección del ambiente. Para lo cual se integran elementos fundamentales como las tradicionales 4 Ps marketing mix; Producto, precio, plaza (distribución) y promoción, se incorpora también 3 Ps de Sostenibilidad Ambiental muy importantes: personas, producción limpia, protección ambiental, articuladas a líneas de actuación: Desarrollo de mercados verdes, producción limpia y consumo sostenible y la inclusión de la variable sostenibilidad en cadenas productivos las mismas que agrupan los elementos de la gestión de marketing, inteligencia de mercados, I+D, etiquetado ambiental, políticas 
ambientales, gestión, difusión y comercialización empresarial consiguiendo desarrollo sostenible. Y por ende mejor calidad de vida.

El objetivo principal de este modelo es Informar para crear conciencia ambiental y cambiar la forma de actuación de empresas y consumidores, desde un razonamiento social del marketing, se tiene en el hecho de que las empresas existen con un propósito: servir a la sociedad, debiendo tener contenido ético y social sus prácticas de marketing-mix.

El modelo de Gestión Estratégica de Marketing Deontológico, pretende generar nuevo conocimiento, contribuir con la responsabilidad social ambiental y sobre todo crear conciencia en las empresas y consumidores de la ciudad de

Ambato - Ecuador y luego ampliar a diferentes entornos nacionales e internacionales.

La gestión estratégica de este modelo está dado por la relación de variables que actúan en contacto con los consumidores es decir el entorno que rodea a la empresa y se lo representa con la siguiente ecuación de regresión:

$$
y=\frac{1}{1+e^{\left(-\beta_{0}-\beta_{1} x_{1}-\beta_{2} x_{2}-\beta_{3} x_{3}-\beta_{4} x_{4}\right)}}
$$

La prueba de Hosmer y Lemeshow de un modelo de regresión logística ( $R L)$. Se dice que el ajuste es aceptable, cuando el valor de la probabilidad predicha $(p)$ es alto, es decir, cercano a 1 de la variable binomial dependiente, mientras que un valor bajo de $\mathrm{p}$ (próximo a 0 ), se asocia con la $Y=0$.

Desde el punto de vista de Kotler, El marketing Mix, es la manera de combinar las variables producto, precio, plaza, promoción para estimular de forma adecuada y permanente los mercados, considerando los efectos que las no controlables ejercen sobre las decisiones de los clientes y prospectos. El marketing para la sostenibilidad, entonces, no busca comercializar productos ecológicos sacrificando las características necesarias para que este satisfaga las necesidades actuales del consumidor, sino que integra todos los subsistemas de la organización para lograr un fin más importante: la garantía de los recursos para

Tabla 3. Esquema teórico del modelo

\begin{tabular}{|c|c|c|c|}
\hline \multicolumn{4}{|c|}{ MODELO DE GESTION DE MARKETING DEONTOLÓGICO } \\
\hline VARIABLES & $\mathbf{x}$ & ELEMENTOS & FINALIDAD \\
\hline \multirow{4}{*}{ Marketing Mix } & \multirow{4}{*}{$\mathrm{X}_{1}$} & Producto & \multirow{4}{*}{$\begin{array}{l}\text { Redefinir las variables del marketing } \\
\text { mix con enfoque de reducción de } \\
\text { contaminación ambiental }\end{array}$} \\
\hline & & Precio & \\
\hline & & Plaza (Distribución) & \\
\hline & & Promoción & \\
\hline \multirow{3}{*}{ Sostenibilidad Ambiental } & \multirow{3}{*}{$\mathrm{X}_{2}$} & Personas & \multirow{3}{*}{$\begin{array}{l}\text { Orientar el marketing a la } \\
\text { responsabilidad social ambiental, } \\
\text { cambiando los patrones de consumo }\end{array}$} \\
\hline & & Producción limpia & \\
\hline & & Protección ambiental & \\
\hline \multirow{3}{*}{ Líneas de acción } & \multirow{3}{*}{$x_{3}$} & Mercados verdes & \multirow{3}{*}{$\begin{array}{c}\text { Mejorar la producción, el consumo } \\
\text { sostenible a lo largo de la cadena } \\
\text { de valor del marketing }\end{array}$} \\
\hline & & $\mathrm{P}+\mathrm{L}$ y consumo sostenible & \\
\hline & & $\begin{array}{c}\text { Sostenibilidad en cadenas } \\
\text { productivas }\end{array}$ & \\
\hline \multirow{7}{*}{ Gestión de Marketing } & \multirow{7}{*}{$X_{4}$} & Inteligencia de mercados & \multirow{7}{*}{$\begin{array}{l}\text { Manejar todos los procesos de } \\
\text { la gestión del marketing con } \\
\text { responsabilidad social ambiental } \\
\text { desde su fabricación hasta la } \\
\text { muerte del producto, tomando en } \\
\text { cuenta todos los factores externos } \\
\text { relevantes }\end{array}$} \\
\hline & & Investigación y desarrollo & \\
\hline & & Etiquetado ambiental & \\
\hline & & Políticas ambientales & \\
\hline & & Gestión empresarial & \\
\hline & & Difusión y publicidad & \\
\hline & & Comercialización & \\
\hline
\end{tabular}


el futuro, mediante el cambio de mentalidad de las empresas y consumidores.

\section{DISCUSIÓN}

El marketing como brazo visible de la empresa, se convierte en el centro de todas las críticas por cuanto su finalidad última es vender una mayor cantidad de producto, en definitiva, generar una mayor rentabilidad. Es necesario entonces la adopción de nuevos conceptos para las variables controlables de marketing: producto, precio, promoción y distribución.

Para el modelo presentado, se define producto: todo bien o servicio que se lo pueda consumir o utilizar sin perjuicio de contaminar el ambiente por medio de sus envases embalajes o residuos del mismo. Con esto se quiere evitar procesos de contaminación, ya que Los datos presentados por PNUMA mencionan que el $70 \%$ del daño ambiental de un producto es debido a su diseño y a los procesos de producción asociados. Por lo tanto, las empresas y los consumidores pueden rediseñar los productos y procesos y mejorar el desempeño ambiental.

Con relación a la variable plaza (distribución) se considera al conjunto de actividades éticas que relaciona la producción con el consumo, así como el conjunto de herramientas usadas para el monitoreo y control del destino del producto y su consumo responsable.

La promoción y publicidad juegan un papel importante en este modelo, por cuanto son el conjunto de actividades que tratan de comunicar los beneficios que reporta el producto, educando y persuadiendo a los consumidores en la responsabilidad social ambiental, utilizando mensajes asertivos, entendidos como la conducta que puede resumirse como un comportamiento mediante el cual defendemos nuestros legítimos derechos sin agredir ni ser agredidos.

En lo que tiene que ver con las Ps de la responsabilidad social ambiental, se menciona: personas; Se define como el compromiso que una persona tiene en relación a la preservación y cuidado del medio ambiente, también se relaciona con los cambios en los comportamientos de compra en los consumidores denominada conciencia ambiental que les da el carácter de Consumidores Ecológicos.

La protección del ambiente en cambio, consiste en el conjunto de medidas que se toman a nivel público y privado para cuidar nuestro hábitat natural, preservándolo del deterioro y la contaminación.
En cuanto a la producción limpia, es una estrategia de carácter preventivo que las empresas pueden aplicar a sus procesos productivos con el objetivo de minimizar los residuos y emisiones en el origen, reduciendo los riesgos para la salud humana y el ambiente y elevando simultáneamente la productividad y la competitividad de la empresa.

Como líneas fundamentales de actuación se tiene, generación de mercados estratégicos verdes; se refiere a la inserción de conceptos ambientales a las actividades tradicionales de mercadeo. Por lo tanto, son mercados donde se transan productos y servicios menos nocivos con el ambiente o derivados del aprovechamiento sostenible de los recursos naturales, siendo necesario indicar que desde la aparición del concepto de desarrollo sostenible se comenzó a generar una visión responsable para con el medio ambiente.

Otra de las líneas es la producción limpia y consumo sostenible, la misma que está relacionada con la aplicación continúa de acciones ambientales preventivas e integradas a procesos, productos y servicios, para aumentar la eficiencia en general y reducir los riesgos para los seres humanos y el ambiente. Se busca entonces que la estrategia de producción más limpia y consumo sostenible involucre el uso de servicios y productos que responden a las necesidades básicas y ofrezcan una mejor calidad de vida, al mismo tiempo que minimizan la contaminación ambiental durante el ciclo de vida del servicio o producto, sin poner en peligro las necesidades de futuras generaciones.

La sostenibilidad en cadenas productivas, no significa necesariamente un cambio radical de todas las actividades organizacionales, más bien requiere que las empresas consideren si pueden dirigir las necesidades del consumidor a una tendencia menos dañina en términos ambientales, implica que todos en la cadena completa del ciclo de vida del producto, desde la cuna hasta la tumba, tienen una responsabilidad y un papel que jugar, tomando en cuenta todos los factores externos relevantes.

\section{CONCLUSIONES}

El modelo de Gestión de marketing deontológico y la responsabilidad social ambiental de consumidores pretende: Concientizar a las empresas y consumidores sobre la importancia de cuidar y proteger el ambiente, Generar mensajes asertivos, Usar racionalmente los recursos, Prevenir impactos ambientales, Reducir el CO2, Modificar patrones de producción y Consumo hacia la sostenibilidad, 
Mejorar la calidad de vida, Lograr la competitividad de empresas.

A manera de conclusión se puede decir que el marketing deontológico pretende incidir en el cambio de conducta de las empresas y los consumidores en el sentido de cuidar y proteger el ambiente

El subsistema marketing constituye un eje transversal en la proyección de la sostenibilidad ambiental, la generación de la ética y valores, entendiéndose, como una postura o filosofía que se debe adopta en las empresas y los consumidores de Ambato-Ecuador.

El aporte que puede dar el marketing deontológico para el desarrollo sostenible no es solamente un conjunto de técnicas destinadas a diseñar y comercializar productos menos perjudiciales para el entorno natural; es, también, una forma de gestión ambiental, un cambio de manera de pensar, actuar y concebir el marketing en los tres ejes, la empresa el consumidor y el ambiente.

Para conseguir el propósito planteado en la ONU sobre desarrollo sostenible, las empresas deben rediseñar ofertas comerciales que permitan satisfacer las necesidades presentes de los consumidores, sin comprometer la capacidad de las necesidades futuras de las próximas generaciones.

\section{REFERENCIAS BIBLIOGRÁFICAS}

[1] Albardiaz, M. (1998). El Comportamiento de Compra Ecológica. Pamplona: Editorial Agricultura Ecológica.

[2] Braidot, N. (1996). Marketing total. Argentina. 5ta edición ampliada. Macchi Grupo Editor S. A

[3] Cannon, T. (1994). La Responsabilidad Social de la Empresa. Barcelona: Ediciones Focio.

[4] Garcia Roca, J. (1994). Desafíos Pendientes para la Década de los 90" en De Cara al Tercer Milenio. Lecciones y Desafíos, 36-55.

[5] Kotler, P. (1999). El Marketing Según Kotler, Cómo Crear, ganar y dominar mercados. Buenos Aires: Paidos Empresa.

[6] INEC, I. N. (2010). Censo de Población y Vivienda. Obtenido de www.ecuadorencifras.ec.

[7] Molina, C. (2010). El Comportamiento Financiero de las Empresas Socialmente Responsables. Europa: Investigación Europea,22.

[8] SENPLADES. (2013-2017). Plan Naciona del Buen Vivir. Quito: Ecuador.

[9] Stanton, W. (2000). Fundamentos de Marketing. Colorado: Mc Graw-Hill.

[10] PNUMA, M. d. (enero de 2009). Producción Más Lmpia. Obtenido de ww.pnuma.org/industrial/ 